\title{
Reverse Mathematics and Well-ordering Principles
}

\author{
Michael Rathjen and Andreas Weiermann ${ }^{1}$ \\ Department of Pure Mathematics \\ University of Leeds \\ Leeds, LS2 9JT, UK \\ Department of Pure Mathematics and Computer Algebra \\ Ghent University \\ Krijgslaan 281 - Gebouw S22, B9000 Gent, Belgium
}

\begin{abstract}
The paper is concerned with generally $\Pi_{2}^{1}$ sentences of the form "if $X$ is well ordered then $f(X)$ is well ordered", where $f$ is a standard proof theoretic function from ordinals to ordinals. It has turned out that a statement of this form is often equivalent to the existence of countable coded $\omega$-models for a particular theory $T_{f}$ whose consistency can be proved by means of a cut elimination theorem in infinitary logic which crucially involves the function $f$. To illustrate this theme, we shall focus on the well-known $\varphi$-function which figures prominently in so-called predicative proof theory. However, the approach taken here lends itself to generalization in that the techniques we employ can be applied to many other proof-theoretic functions associated with cut elimination theorems. In this paper we show that the statement "if $X$ is well ordered then $\varphi X 0$ is well ordered" is equivalent to $\mathbf{A T R}_{0}$. This was first proved by Friedman, Montalban and Weiermann [7] using recursion-theoretic and combinatorial methods. The proof given here is proof-theoretic, the main techniques being Schütte's method of proof search (deduction chains) [13], generalized to $\omega$ logic, and cut elimination for infinitary ramified analysis.
\end{abstract}

Key words: reverse mathematics, well ordering principles, Schütte deduction chains, countable coded $\omega$-model, ATR $_{0}$

PACS: 03B30, 03F05, 03F15, 03F35 03F35

Email address: rathjen@maths.leeds.ac.uk, weiermann@cage.ugent. be (Michael Rathjen and Andreas Weiermann).

1 Research of both authors supported by Royal Society International Joint Projects award 2006/R3. 


\section{Introduction}

The larger project broached in this paper is a form of reverse mathematics for $\Pi_{2}^{1}$ statements of the shape

$\mathbf{W O P}(f) \quad$ "if $X$ is well ordered then $f(X)$ is well ordered"

where $f$ is a standard proof theoretic function from ordinals to ordinals. There are by now several examples of functions $f$ where the statement $\mathbf{W O P}(f)$ has turned out to be equivalent to one of the theories of reverse mathematics over a weak base theory (usually $\mathbf{R} \mathbf{C} \mathbf{A}_{0}$ ). The first example is due to Girard [8].

Theorem 1.1 (Girard 1987) Let $\mathbf{W O}(\mathfrak{X})$ express that $\mathfrak{X}$ is a well ordering. Over $\mathbf{R C A}_{0}$ the following are equivalent:

(i) Arithmetic Comprehension

(ii) $\forall \mathfrak{X}\left[\mathbf{W O}(\mathfrak{X}) \rightarrow \mathbf{W O}\left(2^{\mathfrak{X}}\right)\right]$.

Recently two new results appeared in preprints $[10,7]$. These result give characterizations of the form (1) for the theories $\mathbf{A C A}_{0}^{+}$and $\mathbf{A} \mathbf{T} \mathbf{R}_{0}$, respectively, in the form of familiar proof-theoretic functions. $\mathbf{A C A}_{0}^{+}$denotes the theory $\mathbf{A C A}_{0}$ augmented by an axiom asserting that for any set $X$ the $\omega$-th jump in $X$ exists while $\mathbf{A T R}_{0}$ asserts the existence of sets constructed by transfinite iterations of arithmetical comprehension. $\alpha \mapsto \varepsilon_{\alpha}$ denotes the usual $\varepsilon$ function while $\varphi$ stands for the two-place Veblen function familiar from predicative proof theory (cf. [13]). More detailed descriptions of $\mathbf{A T R}_{0}$ and the function $\mathfrak{X} \mapsto \varphi \mathfrak{X} 0$ will be given shortly. Definitions of the familiar subsystems of reverse mathematics can be found in [15].

Theorem 1.2 (Montalban, Marcone, 2007) Over $\mathbf{R C A}_{0}$ the following are equivalent:

(i) $\mathrm{ACA}_{0}^{+}$

(ii) $\forall \mathfrak{X}\left[\mathbf{W O}(\mathfrak{X}) \rightarrow \mathbf{W O}\left(\varepsilon_{\mathfrak{X}}\right)\right]$.

Theorem 1.3 (Friedman, Montalban, Weiermann 2007) Over $\mathbf{R C A}_{0}$ the following are equivalent:

(i) $\mathbf{A T R}_{0}$

(ii) $\forall \mathfrak{X}[\mathbf{W O}(\mathfrak{X}) \rightarrow \mathbf{W O}(\varphi \mathfrak{X} 0)]$. 
The proof of Theorem 1.3 uses rather sophisticated recursion-theoretic results about linear orderings and is quite combinatorial. Theorem 1.3 uses a result of Steel's [16] about descending sequences of degrees which states that if $Q \subseteq$ $\operatorname{Pow}(\omega) \times \operatorname{Pow}(\omega)$ is arithmetic, then there is no sequence $\left\{A_{n} \mid n \in \omega\right\}$ such that (a) for every $n, A_{n+1}$ is the unique set such that $Q\left(A_{n}, A_{n+1}\right)$, (b) for every $n, A_{n+1}^{\prime} \leq_{T} A_{n}$.

For a proof theorist, theorems 1.2 and 1.3 bear a striking resemblance to cut elimination theorems for infinitary logics. This prompted the first author of this paper to look for proof-theoretic ways of proving these results. The hope was that this would also unearth a common pattern behind them and possibly lead to more results of this kind. The project commenced with [2] where a purely proof-theoretic proof of Theorem 1.2 was presented. In this paper we shall give a new proof of Theorem 1.3. It is principally proof-theoretic, the main techniques being Schütte's method of proof search (deduction chains) [13] and cut elimination for ramified analysis. The general pattern, of which this paper provides a second example, is that a statement $\mathbf{W O P}(f)$ is often equivalent to a familiar cut elimination theorem for an infinitary logic which in turn is equivalent to the assertion that every set is contained in an $\omega$-model of a certain theory $T_{f}$.

To guide the reader through the paper we shall briefly sketch the main parts of the proof of Theorem 1.3, i.e., that (ii) implies (i). We start with the observation that $\mathbf{A} \mathbf{T R} \mathbf{R}_{0}$ can be be axiomatized over $\mathbf{A C A}_{0}$ via a single sentence of the form $\forall X\left(\mathbf{W O}\left(<_{X}\right) \rightarrow \forall Z \exists Y B_{0}(X, Y, Z)\right)$ where $B_{0}(X, Y, Z)$ is an arithmetical formula (cf. Lemma 3.2). Thus to verify $\mathbf{A T R}_{0}$ it suffices to show that for every well-ordering $<_{Q}$ there exists an $\omega$-model of $\mathbb{M}$ of $\mathbf{A C A} \mathbf{A}_{0}$ which contains $Q$ such that $\mathbb{M} \models \forall Z \exists Y B_{0}(X, Y, Z)$. To find $\mathbb{M}$ we employ Schütte's method of proof search from [13, II $\S 4]$, which he used to prove the completeness theorem for first order logic (cf. [13, Theorem 5.7]). The method has to be extended to $\omega$-logic, though. Rather than working in the Schütte calculus of positive and negative forms we work in a Gentzen sequent calculus with finite sets of formulas called sequents. Let $C$ be a sentence that axiomatizes arithmetic comprehension and let $D_{Q}(n)$ be the formula $n \in Q$ if the latter formula is true and $n \notin Q$ otherwise. The main idea is to start with the sequent $\left\{\neg \forall Z \exists Y B_{0}(Q, Y, Z), \neg C, \neg D_{Q}(0)\right\}$ and systematically apply the rules of $\omega$-logic for the second order sequent calculus backwards, giving rise to a tree of sequents $\mathcal{D}_{Q}$. One also has to add the formula $\neg D_{Q}(n)$ to all sequents generated in this way after $n$ steps.

There are two possible outcomes. If the tree $\mathcal{D}_{Q}$ is not well-founded then it contains an infinite path $\mathbb{P}$. Now define a set $M$ via

$$
(M)_{i}=\left\{n \mid n \notin U_{i} \text { occurs in } \mathbb{P}\right\}
$$


and let $\mathbb{M}=\left(\mathbb{N} ;\left\{(M)_{i} \mid i \in \mathbb{N}\right\},+, \cdot, 0,1,<\right)$. For a formula $F$, let $F \in \mathbb{P}$ mean that $F$ occurs in $\mathbb{P}$, i.e. $F \in \Gamma$ for some $\Gamma \in \mathbb{P}$. Let $U_{0}, U_{1}, U_{2}, \ldots$ be an enumeration of the free set variables. For the assignment $U_{i} \mapsto(M)_{i}$ one can then show that $F \in \mathbb{P} \Rightarrow \mathbb{M} \models \neg F$. Whence $\mathbb{M}$ is an $\omega$-model of ACA and $\mathbb{M}=\forall Z \exists Y B_{0}(Q, Y, Z)$. Also note that $(M)_{0}=Q$, thus $Q$ is in $\mathbb{M}$.

The other conceivable outcome is that $\mathcal{D}_{Q}$ is well-founded, i.e. all paths in $\mathcal{D}_{Q}$ are finite, and thus every maximal path ends in a sequent which contains a basic axiom. In other words $\mathcal{D}_{Q}$ is proof tree and the Kleene-Brouwer ordering of this tree is some well-ordering $\tau$. The crucial step to perform next is viewing $\mathcal{D}_{Q}$ as a skeleton of proof tree in infinitary ramified analysis, dubbed $\mathbf{R} \mathbf{A}^{*}$ in [13]. In actuality $\mathcal{D}_{Q}$ can be viewed as the skeleton of a proof of the empty sequent in $\mathbf{R A}^{*}$. As we can remove all cuts in this proof we end up with a cut free proof of the empty sequent. But this is impossible, and therefore $\mathcal{D}_{Q}$ cannot be well-founded. To be able to carry out the removal of all cuts we require crucial help from arithmetical transfinite induction, roughly up to the ordinal $\varphi \tau 0$, hence this is where the principle $\forall \mathfrak{X}[\mathbf{W O}(\mathfrak{X}) \rightarrow \mathbf{W O}(\varphi \mathfrak{X} 0)]$ enters the stage in showing Theorem 1.3(i).

\section{$2 \quad$ The ordering $\varphi \mathfrak{X} 0$}

Via simple coding procedures, countable well-orderings and functions on them can be expressed in the language of second order arithmetic, $\mathrm{L}_{2}$. Variables $X, Y, Z, \ldots$ are supposed to range over subsets of $\mathbb{N}$. Using an elementary injective pairing function $\langle$,$\left.\rangle (e.g. \langle n, m\rangle:=(n+m)^{2}+n+1\right)$, every set $X$ encodes a sequence of sets $(X)_{i}$, where $(X)_{i}:=\{m \mid\langle i, m\rangle \in X\}$. We also adopt from [15], II.2 the method of encoding a finite sequence $\left(n_{0}, \ldots, n_{k-1}\right)$ of natural numbers as a single number $\left\langle n_{0}, \ldots, n_{k-1}\right\rangle$.

Definition 2.1 Every set of natural numbers $Q$ can be viewed as encoding a binary relation $<_{Q}$ on $\mathbb{N}$ via $n<_{Q} m$ iff $\langle n, m\rangle \in Q$. The field of $Q, \operatorname{fld}(Q)$ is the set $\left\{n \mid \exists m\left[n<_{Q} m \vee m<_{Q} n\right]\right\}$.

We say that $Q$ is a well-ordering if $<_{Q}$ is a well-ordering, that is $<_{Q}$ is a linear ordering of its field and every non-empty subset $U$ of $\operatorname{fld}(Q)$ has a $<_{Q}$-least element.

Definition 2.2 Let $Q$ be a linear ordering with least element $0_{Q}$. Let $\varphi u a:=$ $\langle 0,\langle u, a\rangle\rangle, \mathbf{H}:=\{\varphi u a \mid u, a \in \mathbb{N}\}, \mathbf{h}(\varphi u a)=u$ and $\mathbf{h}(b)=0_{Q}$ if $b \notin \mathbf{H}$.

We introduce the ordering $\varphi Q 0$ by inductively defining its field $\operatorname{fld}(\varphi Q 0)$ and the ordering $<_{\varphi Q 0}$ : 
(1) $0 \in \operatorname{fld}(Q)$.

(2) $0<_{\varphi Q 0} \alpha$ if $\alpha \in \operatorname{fld}(\varphi Q 0)$ and $\alpha \neq 0$.

(3) $\varphi u \alpha \in \operatorname{fld}(\varphi Q 0)$ if $u \in \operatorname{fld}(Q), \alpha \in \operatorname{fld}(\varphi Q 0)$ and $\mathbf{h}(\alpha) \leq_{Q} u$.

(4) If $\alpha_{1}, \ldots, \alpha_{n} \in \operatorname{fld}(\varphi Q 0) \cap \mathbf{H}, n>1$ and $\alpha_{n} \leq_{\varphi Q 0} \cdots \leq_{\varphi Q 0} \alpha_{1}$, then

$$
\alpha_{1}+\ldots+\alpha_{n} \in \operatorname{fld}(\varphi Q 0)
$$

where $\alpha_{1}+\ldots+\alpha_{n}:=\left\langle 1,\left\langle\alpha_{1}, \ldots, \alpha_{n}\right\rangle\right\rangle$.

(5) If $\alpha_{1}+\ldots+\alpha_{n}, \beta_{1}+\ldots+\beta_{m} \in \operatorname{fld}(\varphi Q 0)$, then

$$
\begin{aligned}
& \alpha_{1}+\ldots+\alpha_{n}<_{\varphi Q 0} \beta_{1}+\ldots+\beta_{m} \text { iff } \\
& n<m \wedge \forall i \leq n \alpha_{i}=\beta_{i} \text { or } \\
& \exists i \leq \min (n, m)\left[\alpha_{i}<_{\varphi Q 0} \beta_{i} \wedge \forall j<i \alpha_{j}=\beta_{j}\right] .
\end{aligned}
$$

(6) If $\alpha_{1}+\ldots+\alpha_{n} \in \operatorname{fld}(\varphi Q 0), \varphi u \beta \in \operatorname{fld}(\varphi Q 0)$ and $\varphi u \beta \leq_{\varphi Q 0} \alpha_{1}$ then $\varphi u \beta<_{\varphi Q 0} \alpha_{1}+\ldots+\alpha_{n}$.

(7) If $\alpha_{1}+\ldots+\alpha_{n} \in \operatorname{fld}(\varphi Q 0), \varphi u \beta \in \operatorname{fld}(\varphi Q 0)$ and $\alpha_{1}<_{\varphi Q 0} \varphi u \beta$ then $\alpha_{1}+\ldots+\alpha_{n}<_{\varphi Q 0} \varphi u \beta$.

(8) If $\varphi u \alpha, \varphi v \beta \in \operatorname{fld}(\varphi Q 0)$, then

$$
\begin{aligned}
\varphi u \alpha<_{\varphi Q 0} \varphi v \beta \text { iff } & u<_{Q} v \wedge \alpha<_{\varphi Q 0} \varphi v \beta \text { or } \\
& u=v \wedge \alpha<_{\varphi Q 0} \beta \text { or } \\
& v<_{Q} u \wedge \varphi u \alpha<_{\varphi Q 0} \beta .
\end{aligned}
$$

\section{Lemma $2.3\left(\mathrm{RCA}_{0}\right)$}

(i) If $Q$ is a linear ordering then so is $\varphi Q 0$.

(ii) $\varphi Q 0$ is elementary recursive in $Q$.

\section{The theory ATR $_{0}$}

Definition 3.1 Let $A(u, Y)$ be any formula. Define $H_{A}(X, Y)$ to be the formula which says that $<_{X}$ is a linear ordering and that $Y$ is equal to the set of pairs $\langle n, j\rangle$ such that $j$ is in the field of $<_{X}$ and $A\left(n, Y^{j}\right)$ where $Y^{j}=\left\{\langle m, i\rangle \mid i<_{X} j \wedge\langle m, i\rangle \in Y\right\}$. Intuitively $H_{A}(X, Y)$ says that $Y$ is the result of iterating $A$ along $<_{X}$. 
$\mathbf{A T R}_{0}$ is the formal system in the language of second order arithmetic whose axioms consist of $\mathbf{A C A}_{0}$ plus all instances of

$$
\forall X\left(\mathbf{W O}\left(<_{X}\right) \rightarrow \exists Y H_{A}(X, Y)\right)
$$

where $A$ is arithmetical.

Lemma 3.2 $\mathbf{A T R}_{0}$ can be axiomatized over $\mathbf{A C A}_{0}$ via a single sentence

$$
\forall X\left(\mathbf{W O}\left(<_{X}\right) \rightarrow \forall Z \exists Y B_{0}(X, Y, Z)\right)
$$

where $B_{0}(X, Y, Z)$ is of the form $H_{A}(X, Y)$ for some arithmetical formula $A(u, Y, Z)$ with all free variables exhibited.

Proof: This is a standard result. One could for instance take $B_{0}(X, Y, Z)$ to mean that $Y$ is obtained from $Z$ by iterated the Turing jump operation along $<_{X}$ starting with $Z$; so $A(u, Y, Z)$ would actually be a $\Sigma_{1}^{0}$ (complete) formula. Another (shorter and citable) way of showing this is to use the fact that $\mathbf{A T R}_{0}$ is equivalent over $\mathbf{R C A} \mathbf{A}_{0}$ to the statement that every two well-orderings are comparable (see [15], Theorem V.6.8). The proof of the latter statement in $\mathbf{A T R}_{0}$ just requires an instance $H_{A}$ of said form (see the proof of [15] Lemma V.2.9).

Definition 3.3 Let $T$ be a theory in the language of second order arithmetic, $\mathrm{L}_{2}$. A countable coded $\omega$-model of $T$ is a set $W \subseteq \mathbb{N}$, viewed as encoding the $\mathrm{L}_{2}$-model

$$
\mathbb{M}=(\mathbb{N}, \mathcal{S},+, \cdot, 0,1,<)
$$

with $\mathcal{S}=\left\{(W)_{n} \mid n \in \mathbb{N}\right\}$ such that $\mathbb{M} \models T$.

This definition can be made in $\mathbf{R C A} \mathbf{A}_{0}$ (see [15], Definition VII.2).

We write $X \in W$ if $\exists n X=(W)_{n}$.

\section{Main Theorem}

The main result we want to prove is the following.

Theorem 4.1 $\mathbf{R C A}_{0}+\forall \mathfrak{X}[\mathbf{W O}(\mathfrak{X}) \rightarrow \mathbf{W O}(\varphi \mathfrak{X} 0)]$ proves $\mathbf{A T R}_{0}$.

A central ingredient of the proof will be a method of proof search (deduction chains) pioneered by Schütte [13]. 


\subsection{Deduction chains in $\omega$-logic}

Definition 4.2(i) Let $U_{0}, U_{1}, U_{2}, \ldots$ be an enumeration of the free set variables of $\mathrm{L}_{2}$. For a closed term $t$, let $t^{\mathbb{N}}$ be its numerical value. We shall assume that all predicate symbols of the language $\mathrm{L}_{2}$ are symbols for primitive recursive relations. $\mathrm{L}_{2}$ contains predicate symbols for the primitive recursive relations of equality and inequality and possibly more (or all) primitive recursive relations. If $R$ is a predicate symbol in $\mathrm{L}_{2}$ we denote by $R^{\mathbb{N}}$ the primitive recursive relation it stands for. If $t_{1}, \ldots, t_{n}$ are closed terms the formula $R\left(t_{1}, \ldots, t_{n}\right)\left(\neg R\left(t_{1}, \ldots, t_{n}\right)\right)$ is said to be true if $R^{\mathbb{N}}\left(t_{1}^{\mathbb{N}}, \ldots, t_{n}^{\mathbb{N}}\right)$ is true (is false).

(ii) Henceforth a sequent will be a finite set of $\mathrm{L}_{2}$-formulas without free number variables.

(iii) A sequent $\Gamma$ is axiomatic if it satisfies at least one of the following conditions:

(1) $\Gamma$ contains a true literal, i.e. a true formula of either form $R\left(t_{1}, \ldots, t_{n}\right)$ or $\neg R\left(t_{1}, \ldots, t_{n}\right)$, where $R$ is a predicate symbol in $\mathrm{L}_{2}$ for a primitive recursive relation and $t_{1}, \ldots, t_{n}$ are closed terms.

(2) $\Gamma$ contains formulas $s \in U$ and $t \notin U$ for some set variable $U$ and terms $s, t$ with $s^{\mathbb{N}}=t^{\mathbb{N}}$.

(iv) A sequent is reducible or a redex if it is not axiomatic and contains a formula which is not a literal.

Definition 4.3 For $Q \subseteq \mathbb{N}$ define

$$
D_{Q}(n)= \begin{cases}\bar{n} \in U_{0} & \text { if } n \in Q \\ \bar{n} \notin U_{0} & \text { otherwise }\end{cases}
$$

For the proof of Theorem 4.1 it is convenient to have a finite axiomatization of arithmetic comprehension.

Lemma 4.4 $\mathbf{A C A}_{0}$ can be axiomatized via a single $\Pi_{2}^{1}$ sentence $\forall X C(X)$.

Proof: [15], Lemma VIII.1.5.

Definition 4.5 Let $<_{Q}$ be a well-ordering. Let $B\left(U_{i}\right)$ be the formula $\exists Y B_{0}\left(U_{0}, Y, U_{i}\right)$ of Lemma 3.2.

A $Q$-deduction chain is a finite string

$$
\Gamma_{0}, \Gamma_{1}, \ldots, \Gamma_{k}
$$

of sequents $\Gamma_{i}$ constructed according to the following rules: 
(i) $\Gamma_{0}=\neg D_{Q}(0), \neg B\left(U_{0}\right), \neg C\left(U_{0}\right)$.

(ii) $\Gamma_{i}$ is not axiomatic for $i<k$.

(iii) If $i<k$ and $\Gamma_{i}$ is not reducible then

$$
\Gamma_{i+1}=\Gamma_{i}, \neg D_{Q}(i+1), \neg B\left(U_{i+1}\right), \neg C\left(U_{i+1}\right) .
$$

(iv) Every reducible $\Gamma_{i}$ with $i<k$ is of the form

$$
\Gamma_{i}^{\prime}, E, \Gamma_{i}^{\prime \prime}
$$

where $E$ is not a literal and $\Gamma_{i}^{\prime}$ contains only literals.

$E$ is said to be the redex of $\Gamma_{i}$.

Let $i<k$ and $\Gamma_{i}$ be reducible. $\Gamma_{i+1}$ is obtained from $\Gamma_{i}=\Gamma_{i}^{\prime}, E, \Gamma_{i}^{\prime \prime}$ as follows:

(1) If $E \equiv E_{0} \vee E_{1}$ then

$$
\Gamma_{i+1}=\Gamma_{i}^{\prime}, E_{0}, E_{1}, \neg D_{Q}(i+1), \neg B\left(U_{i+1}\right), \neg C\left(U_{i+1}\right) .
$$

(2) If $E \equiv E_{0} \wedge E_{1}$ then

$$
\Gamma_{i+1}=\Gamma_{i}^{\prime}, E_{j}, \neg D_{Q}(i+1), \neg B\left(U_{i+1}\right), \neg C\left(U_{i+1}\right)
$$

where $j=0$ or $j=1$.

(3) If $E \equiv \exists x F(x)$ then

$$
\Gamma_{i+1}=\Gamma_{i}^{\prime}, F(\bar{m}), \neg D_{Q}(i+1), \neg B\left(U_{i+1}\right), \neg C\left(U_{i+1}\right), E
$$

where $m$ is the first number such that $F(\bar{m})$ does not occur in $\Gamma_{0}, \ldots, \Gamma_{i}$.

(4) If $E \equiv \forall x F(x)$ then

$$
\Gamma_{i+1}=\Gamma_{i}^{\prime}, F(\bar{m}), \neg D_{Q}(i+1), \neg B\left(U_{i+1}\right), \neg C\left(U_{i+1}\right)
$$

for some $m$.

(5) If $E \equiv \exists X F(X)$ then

$$
\Gamma_{i+1}=\Gamma_{i}^{\prime}, F\left(U_{m}\right), \neg D_{Q}(i+1), \neg B\left(U_{i+1}\right), \neg C\left(U_{i+1}\right), E
$$

where $m$ is the first number such that $F\left(U_{m}\right)$ does not occur in $\Gamma_{0}, \ldots, \Gamma_{i}$.

(6) If $E \equiv \forall X F(X)$ then

$$
\Gamma_{i+1}=\Gamma_{i}^{\prime}, F\left(U_{m}\right), \neg D_{Q}(i+1), \neg B\left(U_{i+1}\right), \neg C\left(U_{i+1}\right)
$$

where $m$ is the first number such that $m \neq i+1$ and $U_{m}$ does not occur in $\Gamma_{i}$.

The set of $Q$-deduction chains forms a tree $\mathcal{D}_{Q}$ labeled with strings of sequents. We will now consider two cases. 
Case I: $\mathcal{D}_{Q}$ is not well-founded. Then $\mathcal{D}_{Q}$ contains an infinite path $\mathbb{P}$. Now define a set $M$ via

$$
(M)_{i}=\left\{t^{\mathbb{N}} \mid t \notin U_{i} \text { occurs in } \mathbb{P}\right\}
$$

Set $\mathbb{M}=\left(\mathbb{N} ;\left\{(M)_{i} \mid i \in \mathbb{N}\right\},+, \cdot, 0,1,<\right)$.

For a formula $F$, let $F \in \mathbb{P}$ mean that $F$ occurs in $\mathbb{P}$, i.e. $F \in \Gamma$ for some $\Gamma \in \mathbb{P}$.

Claim: Under the assignment $U_{i} \mapsto(M)_{i}$ we have

$$
F \in \mathbb{P} \quad \Rightarrow \quad \mathbb{M} \models \neg F
$$

The Claim will imply that $\mathbb{M}$ is an $\omega$-model of ACA. Also note that $(M)_{0}=Q$, thus $Q$ is in $\mathbb{M}$. The proof of (3) follows by induction on $F$ using Lemma 4.6 below. The upshot of the foregoing is that we can prove Theorem 4.1 under the assumption that $\mathcal{D}_{Q}$ is ill-founded for all sets $Q \subseteq \mathbb{N}$.

Lemma 4.6 Let $Q$ be an arbitrary subset of $\mathbb{N}$ and $\mathcal{D}_{Q}$ be the corresponding deduction tree. Moreover, suppose $\mathcal{D}_{Q}$ is not well-founded. Then $\mathcal{D}_{Q}$ has an infinite path $\mathbb{P} . \mathbb{P}$ has the following properties:

(1) $\mathbb{P}$ does not contain literals which are true in $\mathbb{N}$.

(2) $\mathbb{P}$ does not contain formulas $s \in U_{i}$ and $t \notin U_{i}$ for constant terms $s$ and $t$ such that $s^{\mathbb{N}}=t^{\mathbb{N}}$.

(3) If $\mathbb{P}$ contains $E_{0} \vee E_{1}$ then $\mathbb{P}$ contains $E_{0}$ and $E_{1}$.

(4) If $\mathbb{P}$ contains $E_{0} \wedge E_{1}$ then $\mathbb{P}$ contains $E_{0}$ or $E_{1}$.

(5) If $\mathbb{P}$ contains $\exists x F(x)$ then $\mathbb{P}$ contains $F(\bar{n})$ for all $n$.

(6) If $\mathbb{P}$ contains $\forall x F(x)$ then $\mathbb{P}$ contains $F(\bar{n})$ for some $n$.

(7) If $\mathbb{P}$ contains $\exists X F(X)$ then $\mathbb{P}$ contains $F\left(U_{m}\right)$ for all $m$.

(8) If $\mathbb{P}$ contains $\forall X F(X)$ then $\mathbb{P}$ contains $F\left(U_{m}\right)$ for some $m$.

(9) $\mathbb{P}$ contains $\neg B\left(U_{m}\right)$ for all $m$.

(10) $\mathbb{P}$ contains $\neg C\left(U_{m}\right)$ for all $m$.

(11) $\mathbb{P}$ contains $\neg D_{Q}(m)$ for all $m$.

Proof: Standard.

Corollary 4.7 If $\mathcal{D}_{Q}$ is ill-founded then there exists a countable coded $\omega$ model of $\mathbf{A C A}_{0}$ containing $Q$ which satisfies $\forall Z \exists Y B_{0}(Q, Y, Z)$.

The remainder of the paper will be devoted to ruling out the possibility that, whenever $Q$ is a well-ordering, $\mathcal{D}_{Q}$ can be a well-founded tree. This is the place were cut elimination for the infinitary proof system of ramified analysis, RA* (see [13], part C), enters the stage. In a nutshell the idea is that a well- 
founded $\mathcal{D}_{Q}$ gives rise to a derivation of the empty sequent (contradiction) in RA ${ }^{*}$ which can be ruled by showing cut elimination for $\mathbf{R} \mathbf{A}^{*}$ using transfinite induction up to $\varphi \mathfrak{X} 0$, where $\mathfrak{X}$ is a well ordering not much longer than $Q$. However, to simplify the technical treatment we first introduce an intermediate system $\Delta_{1}^{1}-\mathbf{C R}_{\infty}^{Q}$ based on the $\Delta_{1}^{1}$-comprehension rule and the $\omega$-rule. This theory basically coincides with Schütte's system DA* (see [13], part C). It is not difficult to see that a well-founded $\mathcal{D}_{Q}$ can be viewed as a derivation of the empty sequent in $\Delta_{1}^{1}-\mathbf{C R}_{\infty}^{Q}$. The last step towards reaching a contradiction consists in embedding $\Delta_{1}^{1}-\mathbf{C R}_{\infty}^{Q}$ into $\mathbf{R} \mathbf{A}^{*}$. Here we can basically follow [13] Theorem 22.14.

\subsection{The infinitary calculus $\Delta_{1}^{1}-\mathbf{C R}_{\infty}^{Q}$}

In what follows we fix $Q \subseteq \mathbb{N}$ such that $<_{Q}$ is a well-ordering. In the main, the system $\Delta_{1}^{1}-\mathbf{C R}_{\infty}^{Q}$ is obtained from $\mathbf{A} \mathbf{C A}_{0}$ by adding the $\Delta_{1}^{1}$-comprehension rule, the $\omega$-rule and the basic diagram of $Q$. The language of $\Delta_{1}^{1}-\mathbf{C R}_{\infty}^{Q}$ is the same as that of $\mathbf{A C A}_{0}$ but the notion of formula comes enriched with set terms. Formulas and set terms are defined simultaneously. Literals are formulas. Every set variable is a set term. If $A(x)$ is a formula without set quantifiers (i.e. arithmetical) then $\{x \mid A(x)\}$ is a set term. If $P$ is a set term and $t$ is a numerical term then $t \in P$ and $t \notin P$ are formulas. The other formation rules pertaining to $\wedge, \vee, \forall x, \exists x, \forall X, \exists X$ are as per usual.

We will be working in a Tait-style formalization of the second order arithmetic with formulas in negation normal form, i.e. negations only in front of atomic formulas. Due to the $\omega$-rule there is no need for formulas with free numerical variables. Thus all sequents below are assumed to consist of formulas without free numerical variables.

\section{Axioms of $\Delta_{1}^{1}-\mathbf{C R}_{\infty}^{Q}$}

(i) $\Gamma, L$ where $L$ is a true literal.

(ii) $\Gamma, s \in U, t \notin U$ where $s^{\mathbb{N}}=t^{\mathbb{N}}$.

(iii) $\Gamma, s \in U_{0}$ if $s^{\mathbb{N}} \in Q$.

(iv) $\Gamma, s \notin U_{0}$ if $s^{\mathbb{N}} \notin Q$.

$$
\text { Rules of } \Delta_{1}^{1}-\mathrm{CR}_{\infty}^{Q}
$$


(^) $\frac{\Gamma, A \quad \Gamma, B}{\Gamma, A \wedge B}$

( $) \frac{\Gamma, A_{i}}{\Gamma, A_{0} \vee A_{1}}$ where $i \in\{0,1\}$

(Cut) $\frac{\Gamma, A \quad \Gamma, \neg A}{\Gamma}$

(w) $\frac{\Gamma, F(\bar{n}) \text { for all } n}{\Gamma, \forall x F(x)}$

$\left(\exists_{1}\right) \frac{\Gamma, F(t)}{\Gamma, \exists x F(x)}$

$\left(\forall_{2}\right) \frac{\Gamma, F(P) \text { for all set terms } P}{\Gamma, \forall X F(X)}$

$\left(\exists_{2}\right) \frac{\Gamma, F(P)}{\Gamma, \exists X F(X)}$ where $P$ is a set term.

$\left(\Delta_{1}^{1}\right.$-CR $) \frac{\forall x\left[\forall Y A_{0}(x, Y) \leftrightarrow \exists Y A_{1}(x, Y)\right]}{\Gamma, \exists X \forall x\left[x \in X \leftrightarrow \forall Y A_{0}(x, Y)\right]}$ with $A_{0}, A_{1}$ arithmetical.

$\left(S T_{1}\right) \frac{\Gamma, A(t)}{\Gamma, t \in P}$ where $P$ is the set term $\{x \mid A(x)\}$.

$\left(S T_{2}\right) \frac{\Gamma, \neg A(t)}{\Gamma, t \notin P}$ where $P$ is the set term $\{x \mid A(x)\}$.

$\Delta_{1}^{1}-\mathbf{C R}_{\infty}^{Q}$ is a sequent calculus version of the system $\mathrm{DA}^{*}$ of $[13, \S 20]$. The language of $\mathrm{DA}^{*}$, though, is based on the connectives $\perp, \forall, \rightarrow$ while $\Delta_{1}^{1}-\mathbf{C R}_{\infty}^{Q}$ has the connectives $\wedge, \vee, \forall, \exists, \neg$ and formulas are in negation normal form, i.e. the negation sign appears only in front of atomic formulas. The other main difference is that the deduction system of $\mathrm{DA}^{*}$ is the Schütte calculus of positive and negative forms whereas $\Delta_{1}^{1}-\mathbf{C R}_{\infty}^{Q}$ 's is the Gentzen sequent calculus.

Lemma 4.8 We shall use $\Delta_{1}^{1}-\mathbf{C R}_{\infty}^{Q} \vdash \Gamma$ to convey that the sequent $\Gamma$ is derivable in $\Delta_{1}^{1}-\mathbf{C R}_{\infty}^{Q}$. Pivotal properties of $\Delta_{1}^{1}-\mathbf{C R}_{\infty}^{Q}$ we shall exploit are the following:

(a) $n \in Q \Rightarrow \Delta_{1}^{1}-\mathbf{C R}_{\infty}^{Q} \vdash \bar{n} \in U_{0}$.

(b) $n \notin Q \Rightarrow \Delta_{1}^{1}-\mathbf{C R}_{\infty}^{Q} \vdash \bar{n} \notin U_{0}$.

(c) $\Delta_{1}^{1}-\mathbf{C R}_{\infty}^{Q} \vdash \mathbf{W O}\left(U_{0}\right)$. 
(d) $\Delta_{1}^{1}-\mathbf{C R}_{\infty}^{Q} \vdash \exists Y H_{A}\left(U_{0}, Y\right)$ for all arithmetical formulas $A(u, Y)$ having no other free numerical variables than $u$.

Proof: (a) and (b) are immediate by the axioms (iii) and (iv) of $\Delta_{1}^{1}-\mathbf{C R}_{\infty}^{Q}$.

(c) follows by (outer) transfinite induction on $<_{Q}$, crucially using the $\omega$-rule. This is standard but it seems to be a challenge to find a reference. Via the axioms (iii) and (iv), the role of $Q$ is played in $\Delta_{1}^{1}-\mathbf{C R}_{\infty}^{Q}$ by the variable $U_{0}$. Writing $s \in Q$ and $s<_{Q} t$ for $s \in U_{0}$ and $\langle s, t\rangle \in U_{0}$, respectively, we would like to show that $\Delta_{1}^{1}$-CR $\mathbf{C R}_{\infty}^{Q} \vdash \forall X\left(\operatorname{Prog}_{Q}(U) \rightarrow \forall x x \in X\right)$, where $\operatorname{Prog}_{Q}(U)$ stands for $\forall x\left[\forall y\left(y<_{Q} x \rightarrow y \in U\right) \rightarrow x \in U\right]$. It suffices to show

$$
\Delta_{1}^{1}-\mathbf{C R}_{\infty}^{Q} \vdash \neg \operatorname{Prog}_{Q}(U), \bar{n} \in U
$$

for all $n$ for an arbitrary set variable $U$. To this end we proceed by induction on $Q$. Inductively assume that $\Delta_{1}^{1}-\mathbf{C R}_{\infty}^{Q} \vdash \neg \operatorname{Prog}_{Q}(U), \bar{m} \in U$ holds for all $m<_{Q} n$. If $m<_{Q} n$ is false then $\langle m, n\rangle \notin Q$ and hence $\Delta_{1}^{1}-\mathbf{C R}_{\infty}^{Q} \vdash \neg \bar{m}<_{Q} \bar{n}$. As a result, $\Delta_{1}^{1}-\mathbf{C R}_{\infty}^{Q} \vdash \neg \operatorname{Prog}_{Q}(U), \neg \bar{m}<_{Q} \bar{n}, \bar{m} \in U$ holds for all $m$. Using $(\vee)$ inferences followed by an application of the $\omega$-rule, we get $\Delta_{1}^{1}-\mathbf{C R}_{\infty}^{Q} \vdash$ $\neg \operatorname{Prog}_{Q}(U), \forall y\left(y<_{Q} \bar{n} \rightarrow u \in U\right)$. As $\Delta_{1}^{1}-\mathbf{C R}_{\infty}^{Q} \vdash \bar{n} \notin Q, \bar{n} \in Q$, an inference $(\vee)$ (and weakening) yields

$$
\Delta_{1}^{1}-\mathbf{C R}_{\infty}^{Q} \vdash \neg \operatorname{Prog}_{Q}(U), \forall y\left(y<_{Q} \bar{n} \rightarrow u \in U\right) \wedge \bar{n} \notin Q, \bar{n} \in Q .
$$

Hence via $\left(\exists_{1}\right)$ we arrive at

$$
\Delta_{1}^{1}-\mathbf{C R}_{\infty}^{Q} \vdash \neg \operatorname{Prog}_{Q}(U), \exists x\left[\forall y\left(y<_{Q} \bar{n} \rightarrow u \in U\right) \wedge \bar{n} \notin Q\right], \bar{n} \in Q,
$$

which is the same as $\Delta_{1}^{1}-\mathbf{C R}_{\infty}^{Q} \vdash \neg \operatorname{Prog}_{Q}(U), \bar{n} \in Q$. Thus, by induction on $<_{Q}$, (4) follows.

(d) also follows by transfinite induction on $<_{Q}$ using $\Delta_{1}^{1}$-CR. A reference will be provided in Lemma 4.10 .

We shall need to measure the length of the previous derivations. For (c) and (d) the lengths of those derivations will be "longer" than $Q$, though not "much longer". Let $\tau$ be the ordinal giving the order-type of $Q$. It is easy to cook up a new ordering $Q^{*}$ in an elementary way from $Q$ corresponding to the ordinal $\omega^{2}+\omega \cdot \tau+\omega$ in such a way that $\mathbf{R C A}_{0}$ suffices to prove $\mathbf{W O}(Q) \rightarrow \mathbf{W O}\left(Q^{*}\right)$ (see [8]). The rationale for the choice of $\omega^{2}+\omega \cdot \tau+\omega$ is that it gives us enough elbow room for calibrating the lengths of the foregoing derivations.

From the standing assumption that $Q$ is a well-ordering we get that $Q^{*}$ is a well-ordering, too. 
Definition 4.9 If $\alpha$ is an element of the field of $<_{Q^{*}}$, we use the notation $\Delta_{1}^{1}-\mathbf{C R}_{\infty}^{Q} \vdash^{\alpha} \Gamma$ to convey that the sequent $\Gamma$ is deducible in $\Delta_{1}^{1}-\mathbf{C R}_{\infty}^{Q}$ via a derivation of length $\leq \alpha$. More formally, this relation is defined by recursion on $\alpha$ as follows: $\Delta_{1}^{1}-\mathbf{C R}_{\infty}^{Q} \vdash^{\alpha} \Gamma$ holds if if either $\Gamma$ is an axiom of $\Delta_{1}^{1}-\mathbf{C R}_{\infty}^{Q}$ or $\Gamma$ is the conclusion of a $\Delta_{1}^{1}-\mathbf{C R}_{\infty}^{Q}$-inference with premisses $\left(\Gamma_{i}\right)_{i \in I}$ such that for every $i \in I$ there exists $\beta_{i}<_{Q^{*}} \alpha$ with $\Delta_{1}^{1}-\mathbf{C R}_{\infty}^{Q} \vdash^{\beta_{i}} \Gamma_{i}$.

Lemma 4.10 (1) $\Delta_{1}^{1}-\mathbf{C R}_{\infty}^{Q} \vdash^{0} D_{Q}(n)$ for all $n$ with 0 being the least element of $Q$.

(2) $\Delta_{1}^{1}-\mathbf{C R}_{\infty}^{Q} \vdash^{\alpha} C(U)$ for some $\alpha \in$ field $\left(Q^{*}\right)$ and all free set variables $U$.

(3) $\Delta_{1}^{1}-\mathbf{C R}_{\infty}^{Q} \vdash^{\beta} \mathbf{W O}\left(U_{0}\right)$ for some $\beta \in \operatorname{field}\left(Q^{*}\right)$.

(4) $\Delta_{1}^{1}-\mathbf{C R}_{\infty}^{Q} \mathcal{F}^{\gamma} \exists Y H_{A}\left(U_{0}, Y\right)$ for some $\gamma \in$ field $\left(Q^{*}\right)$ for all arithmetical formulas $A(u, Y)$ having no other free numerical variables than $u$.

(5) $\Delta_{1}^{1}-\mathbf{C R}_{\infty}^{Q} \vdash^{\delta} B(U)$ for some $\delta \in \operatorname{field}\left(Q^{*}\right)$ and all free set variables $U$.

Proof: (1) is an immediate consequence of Lemma 4.8 (a) and (b). (2) follows since the rule $\left(\exists_{2}\right)$ gives arithmetical comprehension. (3) and (4) correspond to Lemma 4.8 (c) and (d), respectively. A detailed proof of (4) amounts to basically the same as that of $[13, \S 21$ Lemma 14] . (5) is an immediate consequence of (4).

Recall that, by Corollary 4.7, there exists a countable coded $\omega$-model of $\mathbf{A C A}_{0}$ containing $Q$ and satisfying $\forall Z \exists Y B_{0}(Q, Y, Z)$ providing $\mathcal{D}_{Q}$ is ill-founded. Now let us assume that $Q$ is a well-ordering and that $\mathcal{D}_{Q}$ is well-founded. Then $\mathcal{D}_{Q}$ can be viewed as a deduction with hidden cuts involving formulas of the shape $\neg B\left(U_{i+1}\right), \neg C\left(U_{i+1}\right)$ and $\neg D_{Q}(i+1)$. Note that by Lemma 4.10, $\Delta_{1}^{1}-\mathbf{C R}_{\infty}^{Q} \vdash^{0} D_{Q}(n), \Delta_{1}^{1}-\left.\mathbf{C R}_{\infty}^{Q}\right|^{\alpha} C(U)$, and $\Delta_{1}^{1}-\left.\mathbf{C R}_{\infty}^{Q}\right|^{\gamma} B(U)$ for some $\alpha, \gamma \in$ field $\left(Q^{*}\right)$. Thus if $\Gamma$ is the sequent attached to a node $\tau$ of $\mathcal{D}_{Q}$ and $\left(\Gamma_{i}\right)_{i \in I}$ is an enumeration of the sequents attached to the immediate successor nodes of $\tau$ in $\mathcal{D}_{Q}$ then the transition

$$
\frac{\left(\Gamma_{i}\right)_{i \in I}}{\Gamma}
$$

can be viewed as a combination of four inferences in $\Delta_{1}^{1}-\mathbf{C R}_{\infty}^{Q}$, the first one being a logical inferences and the other three being cuts. By interspersing $\mathcal{D}_{Q}$ with cuts and adding three cuts with cut formulas $\neg C\left(U_{0}\right), \neg B\left(U_{0}\right)$ and $\neg D_{Q}(0)$ at the bottom we obtain a derivation $\tilde{\mathcal{D}}_{Q}$ in $\Delta_{1}^{1}-\mathbf{C R}_{\infty}^{Q}$ of the empty sequent. Since the preceding line of arguments can be done in $\mathbf{A} \mathbf{C A} \mathbf{A}_{0}$ we arrive at the following: 
Corollary $4.11\left(\mathbf{A C A}_{0}\right)$ If $Q$ is a well-ordering and $\mathcal{D}_{Q}$ is well-founded then there is a derivation $\tilde{\mathcal{D}}_{Q}$ in $\Delta_{1}^{1}-\mathbf{C R}_{\infty}^{Q}$ of the empty sequent.

To finish the paper we thus have to show that the latter is impossible. This we shall do by embedding $\Delta_{1}^{1}-\mathbf{C R}_{\infty}^{Q}$ into a system $\mathbf{R} \mathbf{A}^{\infty}$ defined below. Note that an upper bound for the length of $\tilde{\mathcal{D}}_{Q}$ is provided by $(\alpha+\gamma+\rho) \cdot 4$, where $\rho$ corresponds to the Kleene-Brouwer ordering on $\mathcal{D}_{Q}$.

\section{Ramified Analysis RA $\mathbf{\infty}_{\infty}$}

The theories $\mathbf{R} \mathbf{A}_{\rho}$ are designed to capture Gödel's notion of constructibility restricted to sets of natural numbers. They use ordinal indexed variables $X^{\alpha}, Y^{\alpha}, Z^{\alpha}, \ldots$ for $\alpha<\rho$, with the intended meaning that level 0 variables range over sets definable by numerical quantification, and level $\alpha>0$ variables range over sets definable by numerical quantification and level $<\alpha$ set quantification. The proof-theoretic ordinal of $\mathbf{R A}_{\alpha}$ is $\varphi \alpha 0$. We are interested in an infinitary version of ramified analysis.

Definition 5.1 $\mathbf{R A}{ }^{\infty}$ is basically the same system as $\mathbf{R} \mathbf{A}^{*}$ in $[13, \S 22]$. One difference is that the language of $\mathbf{R A}^{*}$ is based on the connectives $\perp, \forall, \rightarrow$ while $\mathbf{R A}^{\infty}$ has $\wedge, \vee, \forall, \exists, \neg$ and formulas are in negation normal form, i.e. the negation sign appears only in front of atomic formulas. The other difference is that the deduction system of $\mathbf{R} \mathbf{A}^{*}$ is the Schütte calculus of positive and negative forms whereas $\mathbf{R} \mathbf{A}^{\infty}$ 's is the Gentzen sequent calculus.

The formulas of $\mathbf{R A}^{\infty}$ do not have free numerical variables. Literals are formulas of the form $R\left(t_{1}, \ldots, t_{n}\right)$ and $\neg R\left(t_{1}, \ldots, t_{n}\right)$ with $R$ being a symbol for a primitive recursive relation and $t_{1}, \ldots, t_{n}$ being closed numerical terms.

$\mathbf{R A}^{\infty}$ uses ordinal indexed free set variables $U^{\alpha}, V^{\alpha}, W^{\alpha}, \ldots$ and bound set variables $X^{\beta}, Y^{\beta}, Z^{\beta}, \ldots$ with $\beta>0$, where the ordinals are assumed to be elements of some countable well-ordering $R$.

The set terms and formulas together with their levels are generated as follows (cf. $[13, \S 22])$ :

(1) Every literal is a formula of level 0.

(2) Every free set variable $U^{\alpha}$ is a set term of level $\alpha$.

(3) If $P$ is a set term of level $\alpha$ and $t$ is a numerical term, then $t \in P$ and $t \notin P$ are formulas of level $\alpha$.

(4) If $A$ and $B$ are formulas of levels $\alpha$ and $\beta$, then $A \vee B$ and $A \wedge B$ are formulas of level $\max (\alpha, \beta)$.

(5) If $F(0)$ is a formula of level $\alpha$, then $\forall x F(x)$ and $\exists x F(x)$ are formulas of 
level $\alpha$ and $\{x \mid F(x)\}$ is a set term of level $\alpha$.

(6) If $F\left(U^{\beta}\right)$ is a formula of level $\alpha$ and $\beta>0$, then $\forall X^{\beta} F\left(X^{\beta}\right)$ is a formula of level $\max (\alpha, \beta)$.

Definition 5.2 The calculus $\mathbf{R A}_{Q}^{\infty}$

\section{Axioms}

$\Gamma, L$ where $L$ is a true literal.

$\Gamma, s \in U^{\alpha}, t \notin U^{\alpha}$ where $s^{\mathbb{N}}=t^{\mathbb{N}}$.

$\Gamma, s \in U_{0}$ if $s^{\mathbb{N}} \in Q$.

$\Gamma, s \notin U_{0}$ if $s^{\mathbb{N}} \notin Q$.

\section{Rules}

$(\wedge),(\vee),(\omega)$, numerical $(\exists)$ and (Cut) as per usual

$\left(\exists^{\alpha}\right) \frac{\Gamma, F(P)}{\Gamma, \exists X^{\alpha} F\left(X^{\alpha}\right)} \quad P$ set term of level $<\alpha$.

$\left(\forall^{\alpha}\right) \frac{\Gamma, F(P) \text { for all set terms } P \text { of level }<\alpha}{\Gamma, \forall X^{\alpha} F\left(X^{\alpha}\right)}$

$\left(S T_{1}\right) \frac{\Gamma, F(t)}{\Gamma, t \in\{x \mid F(x)\}}$

$\left(S T_{2}\right) \frac{\Gamma, \neg F(t)}{\Gamma, t \notin\{x \mid F(x)\}}$

Definition 5.3 The cut rank of a formula $A$ in $\mathbf{R A}_{Q}^{\infty},|A|$, is defined as follows (cf. $[13, \S 22])$ :

(1) $|L|=0$ for arithmetical literals $L$.

(2) $\left|t \in U^{\alpha}\right|=\left|t \notin U^{\alpha}\right|=\omega \cdot \alpha$

(3) $\left|B_{0} \wedge B_{1}\right|=\left|B_{0} \vee B_{1}\right|=\max \left(\left|B_{0}\right|,\left|B_{1}\right|\right)+1$

(4) $|\forall x B(x)|=|\exists x B(x)|=|t \in\{x \mid B(x)\}|=|t \notin\{x \mid B(x)\}|=|B(0)|+1$ 
(5) $\left|\forall X^{\alpha} A\left(X^{\alpha}\right)\right|=\left|\exists X^{\alpha} A\left(X^{\alpha}\right)\right|=\max \left(\omega \cdot \gamma,\left|A\left(U^{0}\right)\right|+1\right)$

where $\gamma$ is the level of $\forall X^{\alpha} A\left(X^{\alpha}\right)$.

By recursion on $\alpha$ we define the relation $\left.\mathbf{R A}_{Q}^{\infty}\right|_{\rho} ^{\alpha} \Gamma$ as follows: $\left.\mathbf{R} \mathbf{A}_{Q}^{\infty}\right|_{\frac{\alpha}{\rho}} ^{\alpha} \Gamma$ holds if either $\Gamma$ is an axiom of $\mathbf{R} \mathbf{A}_{Q}^{\infty}$ or $\Gamma$ is the conclusion of an $\mathbf{R} \mathbf{A}_{Q}^{\infty}$ inference with premisses $\left(\Gamma_{i}\right)_{i \in I}$ such that for every $i \in I$ there exists $\beta_{i}<\alpha$ with $\mathbf{R A}_{Q}^{\infty} \frac{\beta_{i}}{\rho} \Gamma_{i}$ and, moreover, if this inference is a cut with cut formula $A$ then $|A|<\rho$.

The following three statements are proved in [13] for the system $\mathbf{R A}^{*}$. It is routine to transfer them to $\mathbf{R A}_{Q}^{\infty}$ since cut elimination in a Schütte calculus of positive and negative is closely related to cut elimination in sequent calculi. Moreover, the additional axioms pertaining to $Q$ do not impede the cut elimination process.

Theorem 5.4 (Cut-elimination I)

$$
\left.\mathbf{R A}_{Q}^{\infty} \vdash_{\eta+1}^{\alpha} \Gamma \Rightarrow \mathbf{R A}_{Q}^{\infty}\right|_{\eta} ^{\omega^{\alpha}} \Gamma
$$

Proof: Similar to [13, §22 Lemma 4].

Theorem 5.5 (Cut-elimination II)

$$
\left.\mathbf{R A}_{Q}^{\infty}\right|_{\omega^{\rho}} ^{\alpha} \Gamma \Rightarrow \mathbf{R A}_{Q}^{\infty} \frac{\varphi \rho \alpha}{0} \Gamma
$$

Proof: Similar to [13, Theorem 22.7].

For a formula $F$ of the language of $\Delta_{1}^{1}-\mathbf{C R}_{\infty}^{Q}$ let $F^{\sigma}$ be the result of replacing every bound variable $X$ by $X^{\sigma}$ and every free set variable by a set term of a level $<\sigma$. For $\Gamma=\left\{F_{1}, \ldots, F_{n}\right\}$ let $\Gamma^{\sigma}=\left\{F_{1}^{\sigma}, \ldots, F_{n}^{\sigma}\right\}$.

\section{Theorem 5.6 (Interpretation Theorem)}

$$
\Delta_{1}^{1}-\mathbf{C R}_{\infty}^{Q} \stackrel{\vdash}{\alpha}^{\alpha} \Gamma \quad \Rightarrow \quad \mathbf{R A}_{Q}^{\infty} \stackrel{\omega \cdot \sigma+\omega+\omega \cdot \alpha}{\omega \cdot \sigma} \Gamma^{\sigma}
$$

for all $\sigma$ of the form $\omega^{\alpha} \cdot \beta$ with $\beta \neq 0$.

Proof: This is basically the same as [13, Theorem 22.14].

There are different ways of formalizing infinite deductions in theories like PA. We just mention [14] and [6]. 


\subsection{Finishing the proof of the main Theorem}

Recall that in order to finish the proof of Theorem 4.1 we want to show that $\mathcal{D}_{Q}$ is not well-founded whenever $Q$ is a well-ordering. By Corollary 4.11 , if $Q$ is a well-ordering and $\mathcal{D}_{Q}$ is well-founded then there is a derivation $\tilde{\mathcal{D}}_{Q}$ in $\Delta_{1}^{1}-\mathbf{C R}_{\infty}^{Q}$ of the empty sequent. By the Interpretation Theorem 5.6 we would then get a derivation in $\mathbf{R} \mathbf{A}_{Q}^{\infty}$ of the empty sequent. Using the principle $\mathbf{W O}(\mathfrak{X}) \rightarrow \mathbf{W O}(\varphi \mathfrak{X} 0)$ we can then employ the cut elimination Theorem 5.5 to obtain a cut-free derivation of the empty sequent in $\mathbf{R} \mathbf{A}_{Q}^{\infty}$. But this is impossible.

From Corollary 4.7 we can thus conclude that for every well-ordering $\tilde{Q}$ there exists a countable coded $\omega$-model of $\mathbf{A C A}_{0}$ containing $\tilde{Q}$ and satisfying $\forall Z \exists Y B_{0}(\tilde{Q}, Y, Z)$. From this we would like to infer that for every well-ordering $Q$ and every set $Z_{0}$ there exists a set $Y$ such that $B_{0}\left(\tilde{Q}, Y, Z_{0}\right)$. We can do this by encoding $Q$ and $Z_{0}$ in a well-ordering $\tilde{Q}$ from which $Q$ and $Z_{0}$ can be retrieved in any $\omega$-model of $\mathbf{A C A}_{0}$ containing $\tilde{Q}$. One way of doing this is to define the new ordering $\tilde{Q}$ by letting

$$
\begin{aligned}
\langle n, m\rangle<_{\tilde{Q}}\left\langle n^{\prime}, m^{\prime}\right\rangle \text { iff }[ & {\left[n=n^{\prime}=0 \wedge m<{ }_{\tilde{Q}} m^{\prime}\right] \vee } \\
& {\left[n=n^{\prime}=1 \wedge m, m^{\prime} \in Z_{0} \wedge m<m^{\prime}\right] \vee } \\
& {\left[n=0 \wedge n^{\prime}=1 \wedge m \in \operatorname{field}(Q) \wedge m^{\prime} \in Z_{0}\right] . }
\end{aligned}
$$

Obviously $\tilde{Q}$ is a well-ordering, too, and any $\omega$-model $\mathbb{M}$ of $\mathbf{A C A}_{0}$ containing $\tilde{Q}$ will contain $Z_{0}$ as well. Moreover, $\mathbb{M} \models \exists Y B_{0}\left(\tilde{Q}, Z_{0}\right)$ implies $\mathbb{M} \models \exists Y B_{0}\left(Q, Z_{0}\right)$. Hence, in view of Lemma 3.2, we get $\mathbf{A} \mathbf{T R}_{0}$, thereby finishing the proof of Theorem 4.1.

\section{$6 \quad$ Finishing the proof of Theorem 1.3}

One direction of Theorem 1.3 follows from Theorem 4.1. The other direction is implicit in the proof of [13] Theorem 21.6.

\section{$7 \quad$ Prospectus}

The methodology exemplified in the proof of Theorem 1.3 should have many more applications. Every cut elimination theorem in ordinal-theoretic proof theory potentially encapsulates a theorem of type 1.3. The first author has looked at two more examples and sketched proofs of the pertaining theorems. 
A familiar function from proof theory is the $\Gamma$-function where $\alpha \mapsto \Gamma_{\alpha}$ enumerates the fixed points of the $\varphi$-function. Since the proof of the next result has only been sketched we classify it as a conjecture.

Conjecture 7.1 Over $\mathbf{R C A}_{0}$ the following are equivalent:

(i) $\mathbf{R C A}_{0}+$ Every set $X$ is contained in a countable coded $\omega$-model of $\mathbf{A T R}_{0}$.

(ii) $\forall \mathfrak{X}\left[\mathbf{W O}(\mathfrak{X}) \rightarrow \mathbf{W O}\left(\Gamma_{\mathfrak{X}}\right)\right]$.

The direction (i) $\Rightarrow$ (ii) follows from $[11,4.13,4.16]$.

For an example from impredicative proof theory one would perhaps first turn to the ordinal representation system used for the ordinal analysis of the theory ID $_{1}$ of non-iterated inductive definitions, which can be expressed in terms of the $\theta$-function (cf. [4]). ID $_{1}$ has the same strength as the subsystem of second order arithmetic based on bar induction, BI (cf. [4,5,12]). In Simpson's book the acronym used for $\mathbf{B I}$ is $\Pi_{\infty}^{1}-\mathbf{T I}_{0}$ (cf. [15, §VII.2]). In place of the function $\theta$ we prefer to work with simpler ordinal representations based on the $\psi$-function introduced in [3] or the $\vartheta$-function of [12]. For definiteness we refer to [12]. Given a well-ordering $\mathfrak{X}$, the relativized versions $\vartheta_{\mathfrak{X}}$ and $\psi_{\mathfrak{X}}$ of the $\vartheta_{\text {-function }}$ and the $\psi$-function, respectively, are obtained by adding all the ordinals from $\mathfrak{X}$ to the sets $C_{n}(\alpha, \beta)$ of $[12, \S 1]$ and $C_{n}(\alpha)$ of $[12$, Definition 3.1] as initial segments, respectively. The resulting well-orderings $\vartheta_{\mathfrak{X}}\left(\varepsilon_{\Omega+1}\right)$ and $\psi_{\mathfrak{X}}\left(\varepsilon_{\Omega+1}\right)$ are equivalent owing to [12, Corollary 3.2].

Again, as the following statement has not been buttressed by a complete proof we formulate it as a conjecture.

Conjecture 7.2 Over $\mathbf{R C A}_{0}$ the following are equivalent:

(i) $\mathbf{R C A}_{0}+$ Every set $X$ is contained in a countable coded $\omega$-model of $\mathbf{B I}$.

(ii) $\forall \mathfrak{X}\left[\mathbf{W O}(\mathfrak{X}) \rightarrow \mathbf{W O}\left(\psi_{\mathfrak{X}}\left(\varepsilon_{\Omega+1}\right)\right)\right]$.

\section{References}

[1] Bahareh Afshari: Proof-Theoretic Strengths of Hierarchies of Theories, $\mathrm{PhD}$ thesis, University of Leeds, U.K., 2008.

[2] Bahareh Afshari and Michael Rathjen: Reverse Mathematics and Well-ordering Principles: A pilot study, to appear in: Annals of Pure and Applied Logic.

[3] W. Buchholz: A new system of proof-theoretic ordinal functions, Ann. Pure Appl. Logic 32 (1986) 195-207. 
[4] W. Buchholz, S. Feferman, W. Pohlers, W. Sieg: Iterated inductive definitions and subsystems of analysis (Springer, Berlin, 1981).

[5] W. Buchholz and K. Schütte: Proof theory of impredicative subsystems of analysis (Bibliopolis, Naples, 1988).

[6] H. Friedman and S. Sheard: Elementary descent recursion and proof theory, Annals of Pure and Applied Logic 71 (1995) 1-45.

[7] Harvey Friedman, Antonio Montalban, Andreas Weiermann: Phi function, draft, 2007.

[8] Jean-Yves Girard: Proof Theory and logical complexity, vol 1 (Bibliopolis, Napoli, 1987).

[9] Jeffry L. Hirst: Reverse mathematics and ordinal exponentiation, Annals of Pure and Applied Logic 66 1-18, North-Holland, 1994.

[10] Alberto Marcone, Antonio Montalbán: The epsilon function for computability theorists, draft, 2007.

[11] M. Rathjen: The strength of Martin-Löf type theory with a superuniverse. Part I. Archive for Mathematical Logic 39 (2000) 1-39.

[12] M. Rathjen and A. Weiermann: Proof-theoretic investigations on Kruskal's theorem, Annals of Pure and Applied Logic 60 (1993) 49-88.

[13] Kurt Schütte: Proof Theory, Springer-Verlag, Berlin, Heidelberg, 1977.

[14] H. Schwichtenberg: Proof Theory: Some applications of cut-elimination. In: Handbook of Mathematical Logic (J. Barwise ed.) North Holland 1977, pp. 867-895.

[15] Stephen G. Simpson: Subsystems of Second Order Arithmetic, Springer-Verlag, Berlin, Heidelberg, 1999.

[16] John Steel: Descending sequences of degrees. Journal of Symbolic Logic 40 (1975) 59-61. 